\title{
Respiratory Consequences of N95 Mask during Covid-19 Pandemic- An Observational Study
}

\author{
Rajesh Prabhakar Gaikwad ${ }^{1}$, Akshaya Bhupesh Banodkar², \\ Vaibhavi Pandurang Nandgaonkar ${ }^{3}$, Lynette Custodio Fernandes ${ }^{4}$, \\ Priyanka Ganpatrao Awasare ${ }^{5}$, Manjiree Suryakant Awad 6 , \\ Shraddha Karkhanis ${ }^{7}$
}

${ }^{1}$ Professor and Head of Department, Department of Periodontology, Government Dental College and Hospital, Mumbai 400001.

${ }^{2}$ Associate Professor, ${ }^{3,4,5}$ Post Graduate student , ${ }^{6,7}$ Assistant Professor, Department of Periodontology, Government Dental College and Hospital, Mumbai 400001.

Corresponding Author: Vaibhavi Pandurang Nandgaonkar

\begin{abstract}
Background: Outbreak of novel corona virus has led World Health Organization (WHO) to come up with healthcare guidelines which includes wearing of N95 mask. The aim of this study was to evaluate whether blood oxygen saturation level is affected by wearing of N95 mask for the duration of 4 hours.

Materials and Methods: A total of 90 exam going students were included in this study, who were instructed to wear $\mathrm{N} 95$ mask. Blood oxygen saturation level $\left(\mathrm{SpO}_{2}\right)$ was checked both before and after wearing of N95 mask for a period of 4 hours.

Results: It was observed that, before wearing $\mathrm{N} 95$ mask at $9 \mathrm{am}, \mathrm{SpO}_{2}$ values were $96.64 \pm 0.196$ while at $1 \mathrm{pm}$ after removing N95 mask, the saturation values were 95.68 $\pm 0.235(\mathrm{p}=0.000)$ among all the students. There was a statistically highly significant difference seen for the values between the time intervals $(\mathrm{p}<0.01)$ with higher values at 9 am and lesser at $1 \mathrm{pm}$. On comparing the $\mathrm{SpO}_{2}$ levels among males and females, a statistically nonsignificant difference was seen. Thus, reduction in blood oxygen saturation level can be a major parameter to evaluate the respiratory consequences of N95 mask.

Conclusion: Various adverse effects of N95 mask were quoted in literature but respiratory consequences due to reduction in blood oxygen saturation level can be harmful. Thus, to avoid such consequences, precautionary measures are advised to be taken.
\end{abstract}

Key Words: N95 mask, $\mathrm{SpO}_{2}$, Blood oxygen saturation, COVID-19,

\section{INTRODUCTION}

In late December 2019, number of local health authorities reported clusters of patients with pneumonia of unknown cause, which were epidemiologically linked to a seafood market in Wuhan, China (Zhu et al., 2019). The pathogen, a novel coronavirus (SARS-CoV-2), was identified by local hospital using a surveillance mechanism for "pneumonia of unknown aetiology" ( $\mathrm{Li}$ et al., 2020a; Zhu et al., 2019). On 30 January
2020, the World Health Organization (WHO) declared that CoVID-19 as a "public-health emergency of international concern" $\left(\mathrm{Li}\right.$ et al,2020). ${ }^{[1]}$ The virus outbreak soon evolved into a public health crisis and has spread exponentially to other parts of the world. ${ }^{[2]}$

In this precarious situation, the WHO and CDC has recommended the use of $\mathrm{N} 95$ respirator mask as preventive measure. ${ }^{[3]}$ According to evidence, COVID- 
19 virus, transmits through respiratory droplets and contact routes. ${ }^{[4]}$ Hence the use of masks is recommended, so as to prevent and limit the spread of respiratory viral diseases.

The N95 respirator is a National Institute for Occupational Safety and Health (NIOSH) certified respiratory protection device basically used to decrease the aerosol exposure. The term "N95" indicates that the respirator blocks at least 95 percent of test particles of $300 \mathrm{~nm}^{[5]}$ The FDA describes the N95 respirator as a "respiratory protective device designed to achieve a very close facial fit and very efficient filtration of airborne particles" (N95 Respirators, Surgical Masks, and Face Masks. ${ }^{[6]}$ But there are certain challenges associated with wearing the N95 masks for longer durations, such as nausea, shortness of breath, complaints of visual challenges, headache, light headedness, and difficulty with communication. ${ }^{[7]}$

Blood oxygen level indicates how much oxygen red blood cells are carrying. Blood oxygenation plays an essential role in ensuring that muscles, brain and other organs receive the energy they need to function properly. An observational study, which was carried out amongst 52 surgeons, wearing surgical masks, found a decrease in arterial $\mathrm{O} 2$ saturation from approximately $98 \%$ before surgery to $96 \%$ after surgery, which ranged from 1-4 $\mathrm{h}$ in length. ${ }^{[8]}$ These values though statistically significant, were not clinically important.

Normal pulse oximeter readings usually range from 95 to $100 \%$. Values under $90 \%$ are considered low and indicate the need for supplemental oxygen. This condition is often referred to as hypoxemia, and its symptoms include severe shortness of breath, increased heart rate and chest pain. A study by Rebmann et al, concluded that prolonged use ( $\geq 12 \mathrm{~h}$ ) of masks (N95 respirator or N95 respirator with a surgical mask overlay) is associated with headaches, light -headedness, shortness of breath and decrease in blood oxygen saturation. ${ }^{[7]}$
The primary focus of this study was to evaluate whether blood oxygen saturation level is affected by wearing of N95 mask for the duration of 4 hours.

\section{MATERIAL AND METHODS}

90 students from both sexes, studying at Government Dental College and Hospital, 22-25 years old, non-smokers and without any chronic lung disease were studied by pulse oximetry before and after the course of an examination. They were afebrile, hemodynamically stable, breathing room air, with no previous history of Corona virus infection or any positive contact history.

Disposable sterile valveless N95 masks were distributed among the students and the mask position did not vary during the examination (never below the nose). The same pulse oximeter (Company name) was used to measure the blood oxygen saturation during the study. Participants were encouraged to speak and behave in their usual manner throughout the period of examination. For all measurements, pulse oximeter was applied to the second finger of the right hand. The study was performed for 3 consecutive days. The temperature (in Celsius) and blood oxygen saturation (in percentage) of each student was recorded before wearing a mask (Figure 1). Then students were asked to wear a mask and instructed not to remove it till the end of an examination (approximately $4 \mathrm{hrs}$ ). After 4 hours, again blood oxygen saturation was checked for each student by using the same pulse oximeter (Figure 2). Students were given instructions on how to wear and remove N95 mask with proper sanitization technique.

Data obtained was compiled on a MS Office Excel Sheet (v 2019, Microsoft Redmond Campus, Redmond, Washington, United States). Data was subjected to statistical analysis using Statistical package for social sciences (SPSS v 26.0, IBM). Descriptive statistics like frequencies and percentage for categorical data, Mean \& SD for numerical data has been depicted. 
Rajesh Prabhakar Gaikwad et.al. Respiratory consequences of N95 mask during Covid-19 pandemic-an observational study.

Normality of numerical data was checked using Shapiro-Wilk test \& was found that the data followed a normal curve; hence parametric tests have been used for comparisons. Intra group comparison was done using paired $\mathrm{t}$ test (upto 2 observations). For all the statistical tests, $\mathrm{p}<0.05$ was considered to be statistically significant, keeping $\alpha$ error at $5 \%$ and $\beta$ error at $20 \%$, thus giving a power to the study as $80 \%$.
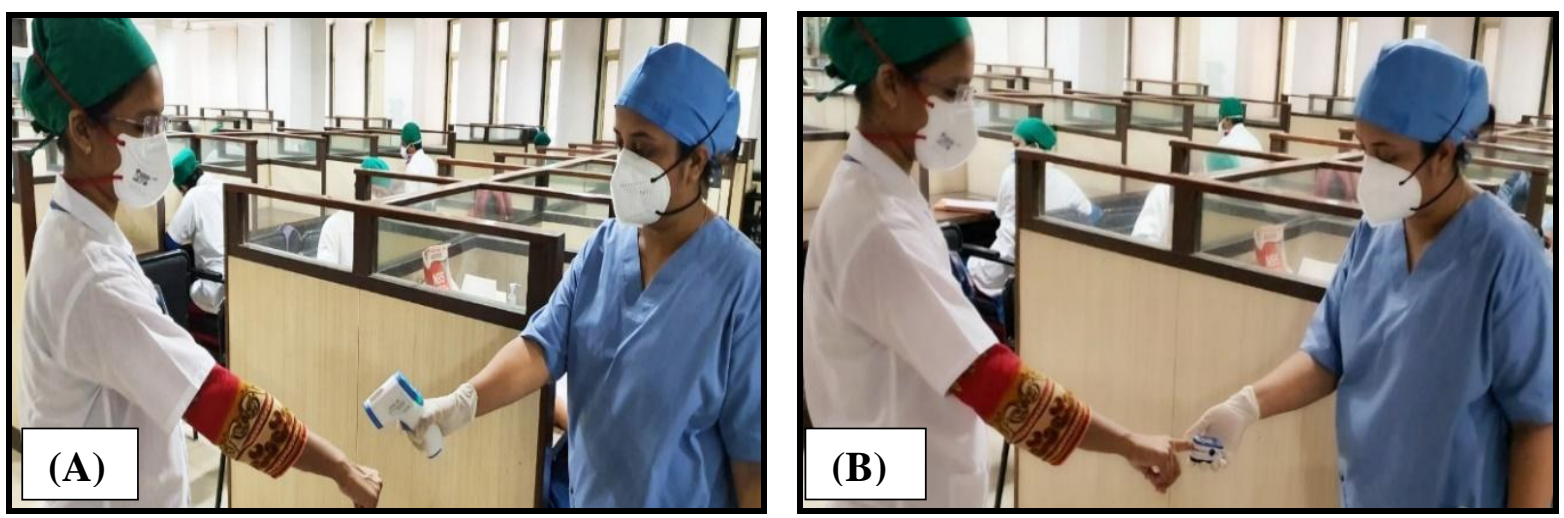

Figure 1. Measuring temperature (A) and blood oxygen saturation level (B) of students
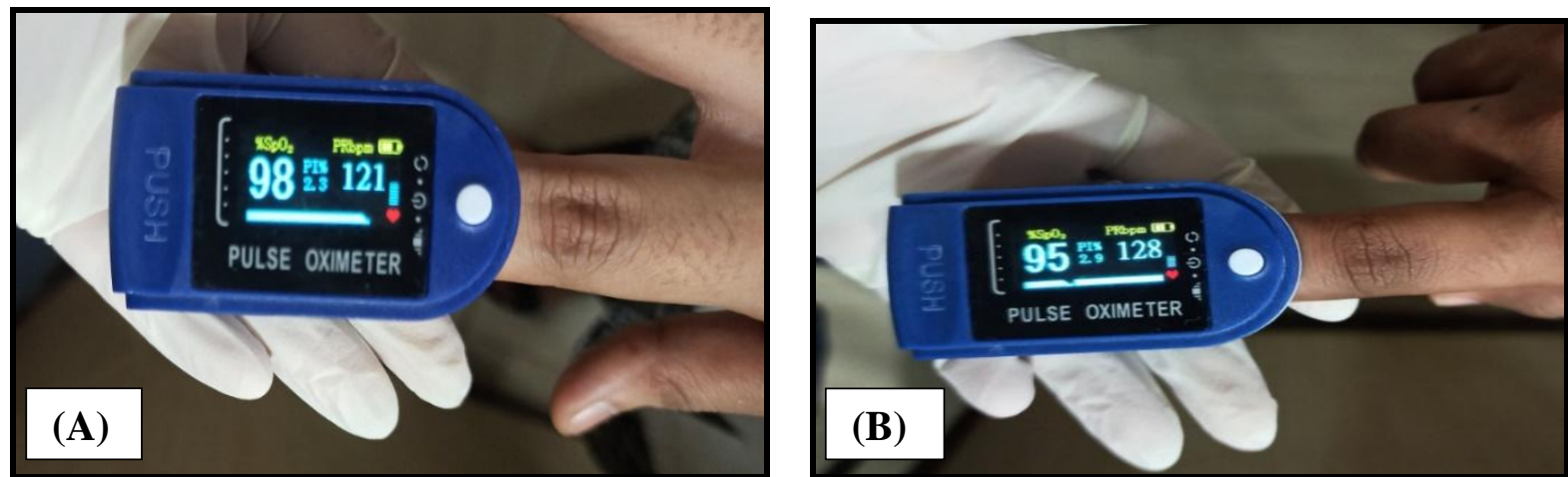

Figure 2. Blood oxygen saturation level of students before (A) and after (B) wearing of mask for 4 hours

\section{RESULTS}

Total 90 students with mean age of 22 years were recruited in this study, among which 60 were females and 30 were males (Figure 3).

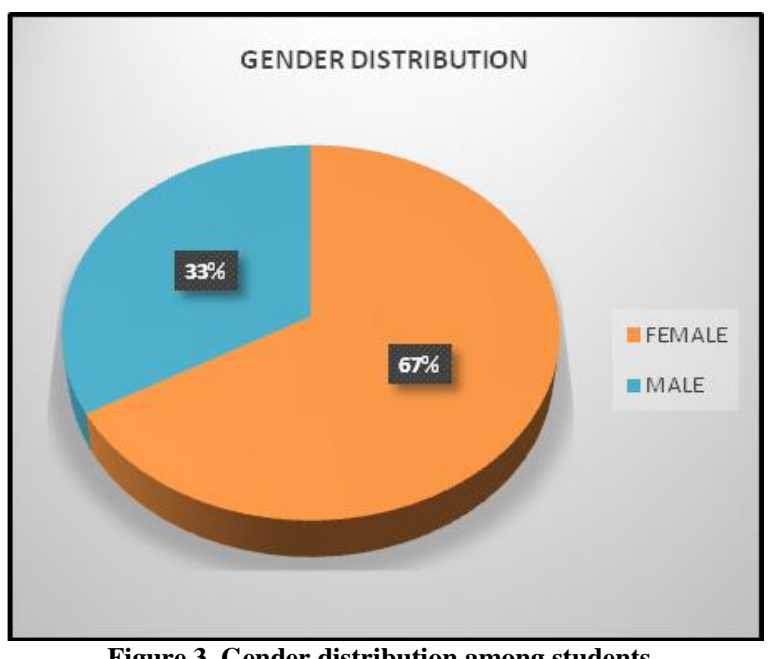

Figure 3. Gender distribution among students

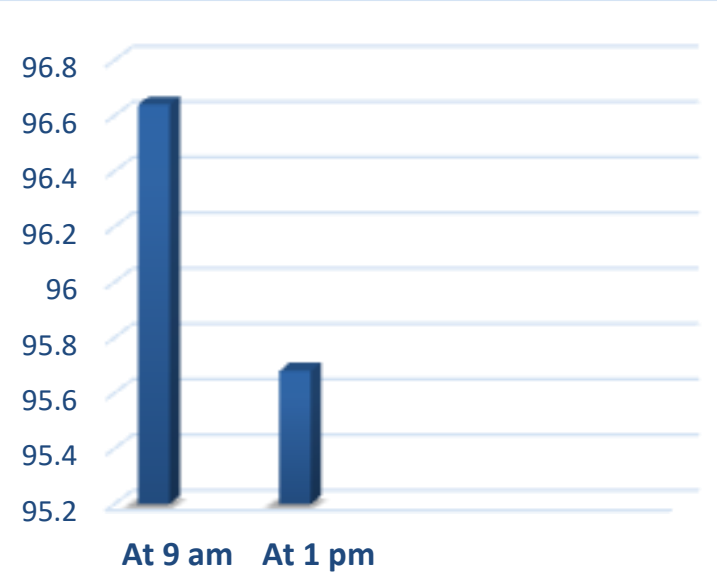

Figure 4. Comparison of $\mathrm{SpO}_{2}$ values of all the students at 9am versus 1 pm

Figure 4, shows comparison of $\mathrm{SpO}_{2}$ values of all students before and after wearing N95 mask. It was observed that, 
Rajesh Prabhakar Gaikwad et.al. Respiratory consequences of N95 mask during Covid-19 pandemic- an observational study.

before wearing N95 mask at 9 am, mean of $\mathrm{SpO}_{2}$ values was $96.64 \pm 0.196$ while at $1 \mathrm{pm}$ after removing $\mathrm{N} 95$ mask, mean of $\mathrm{SpO}_{2}$ values was $95.68 \pm 0.235 \quad(p=0.000)$. A statistically highly significant difference was seen for the values between the time intervals $(\mathrm{p}<0.01)$ with higher values at 9 am and lesser values at $1 \mathrm{pm}$ for all 90 students.

Figure 5, shows comparison of $\mathrm{SpO}_{2}$ values of male students before and after wearing N95 mask. It was observed that, before wearing N95 mask at 9am, mean of $\mathrm{SpO}_{2}$ values was $96.07 \pm 0.279$ while at 1.00 $\mathrm{PM}$ after removing $\mathrm{N} 95$ mask, mean of $\mathrm{SpO}_{2}$ values was $95.37 \pm 0.394(\mathrm{p}=0.000)$. A statistically highly significant difference was seen for the values between the time intervals $(\mathrm{p}<0.01)$ with higher values at 9 am and lesser values at $1 \mathrm{pm}$ for 30 male students.

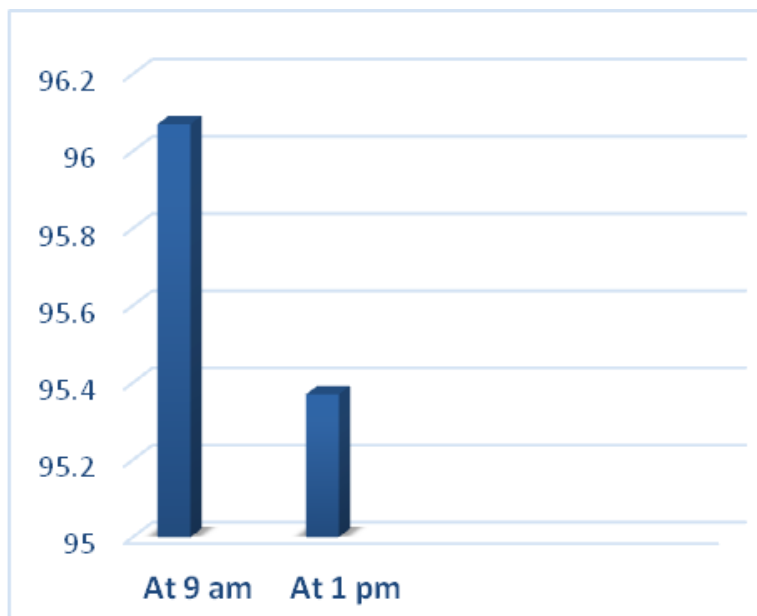

Figure 5. Comparison of $\mathrm{SpO}_{2}$ values of male students at 9am versus $1 \mathrm{pm}$

Figure 6, shows comparison of $\mathrm{SpO}_{2}$ values of all female students before and after wearing N95 mask. It was observed that, before wearing N95 mask at 9 am, mean of $\mathrm{SpO}_{2}$ values was $96.93 \pm 0.251$ while at $1 \mathrm{pm}$ after removing N95 mask, mean of $\mathrm{SpO}_{2}$ values was $95.83 \pm 0.293$ $(\mathrm{p}=0.000)$. A statistically highly significant difference was seen for the values between the time intervals $(\mathrm{p}<0.01)$ with higher values at 9 am and lesser values at $1 \mathrm{pm}$ for 60 female students.

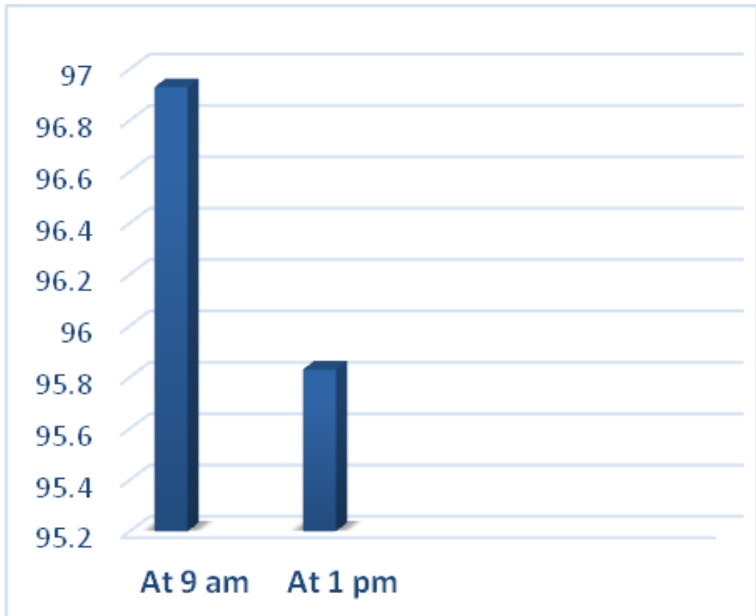

Figure 6. Comparison of $\mathrm{SpO}_{2}$ values of female students at 9am versus $1 \mathrm{pm}$

When $\mathrm{SpO}_{2}$ values of males and females were compared, it was observed that males have 0.362 and females have 0.300 of standard error of mean $(\mathrm{p}=0.423)$. A statistically nonsignificant difference was seen for the values between the groups ( $p>0.05)$. Figure 7 shows the comparison of gender wise difference in $\mathrm{SpO}_{2}$ levels.

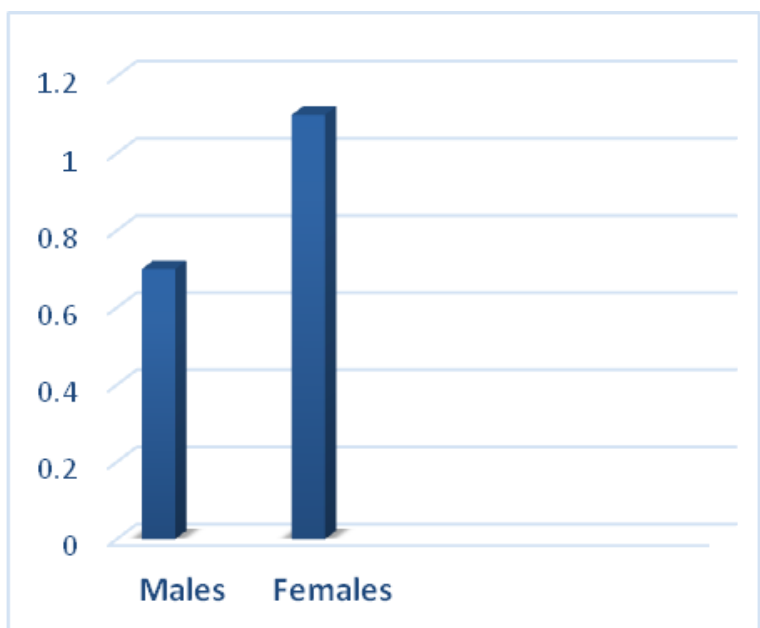

Figure 7. Comparison of gender wise difference in $\mathrm{SpO}_{2}$ levels at 9 am and $1 \mathrm{pm}$

\section{DISCUSSION}

In this study, we observed that there is significant level of reduction in blood oxygen saturation level after wearing N95 mask for a duration of 4 hours. Data showed that both males and females showed significant difference in reduction of blood oxygen saturation level. But when compared blood oxygen saturation level of males 
versus females after 4 hours, nonsignificant difference was observed.

Filtering facepiece respirators (FFRs) are protective devices used in numerous workplaces to reduce airborne particulate exposures. ${ }^{[9]}$ An N95 respirator is a respiratory protective device designed to achieve a very close facial fit and very efficient filtration of airborne particles. The edges of the respirator are designed to form a seal around the nose and mouth. Surgical N95 Respirators are commonly used in healthcare settings and are a subset of N95 Filtering Facepiece Respirators (FFRs), often referred to as N95. ${ }^{[6]} \mathrm{N} 95$ filters are made of several layers of woven synthetic material treated to sustain an electrostatic charge. In the healthcare setting, N95 filters provide adequate protection from most airborne pathogens (e.g., influenza, tuberculosis). ${ }^{[10]}$

An N95 respirator user is going to experience some level of breathing resistance, even though these devices are designed to minimize breathing resistance as much as possible. Enough breathing resistance could result in a reduction in the frequency and depth of breathing due to decreased oxygen level and increased carbon dioxide $\left(\mathrm{CO}_{2}\right)$, known as hypoventilation.

Hypoventilation is a primary cause of significant discomfort while wearing an N95 FFR (Williams 2010). ${ }^{[1]}$ However, studies done by Roberge et al. in 2010, indicated that this hypoventilation did not pose a significant risk to healthcare workers over the course of less than one hour of continuous N95 use. ${ }^{[12]}$ When healthcare workers are working for longer hours without a break while continuously wearing an N95 mask, blood CO2 levels may increase past the 1-hour mark, which could have a significant physiological effect on the wearer (Lim et al., 2006). ${ }^{[13]}$

Some of the known physiological effects of increased concentrations of $\mathrm{CO} 2$ include:

1. Headache;

2. Increased pressure inside the skull;
3. Nervous system changes (e.g., increased pain threshold, reduction in cognition altered judgement, decreased situational awareness, difficulty coordinating sensory or cognitive, abilities and motor activity, decreased visual acuity, widespread activation of the sympathetic nervous system that can oppose the direct effects of $\mathrm{CO} 2$ on the heart and blood vessels);

4. Increased breathing frequency;

5. Increased "work of breathing", which is result of breathing through a filter medium;

6. Cardiovascular effects (e.g., diminished cardiac contractility, vasodilation of peripheral blood vessels);

7. Reduced tolerance to lighter workloads.

To avoid such adverse effects, the balance between the protection afforded by N95 respirators and the burden of these respirators must be met with a plan to mitigate the burden. Healthcare workers are advised to find a safe place to properly take off the respirator to reduce $\mathrm{CO} 2$ build-up and the negative physiological effects associated with it. Breaks during work shifts are vital to individual's health and safety. The potential physiological burden brought on by PPE use can be an unfortunate side effect; however, it can easily be remedied with a little fresh air and proper self-care. Dehydration can be a significant problem when wearing PPE while working in high threat environments. The effects of dehydration may contribute to the experience of the physiological burden such as headache, dizziness, strong sensation of thirst, and reduced cognition. Therefore, healthcare workers must be aware of the need for proper hydration especially if wearing PPE causes significant sweating from heat exposure. A rule of thumb is to drink $1 \mathrm{ml}$ or $1 \mathrm{oz}$ of fluid for every $1 \mathrm{ml}$ or $1 \mathrm{oz}$ of body weight lost. Similarly, one must eat healthy food in part because the calories are needed to provide energy for healthcare workers to continue their work. $^{[14]}$ 
Rajesh Prabhakar Gaikwad et.al. Respiratory consequences of N95 mask during Covid-19 pandemic- an observational study.

Various alternative respirators have been available for the use in order to prevent spread of airborne infections. Elastomeric Respirators (EHMRs) can be one of the alternative to N95 FFRs due to their reusability (Hines et al., 2019). ${ }^{[15]}$ However, the physiological burden on the wearer is more likely to cause anxiety when wearing an EHMR compared to FFRs (Wu et al., 2011). ${ }^{[16]}$ The increased breathing resistances found in EHMRs can result in a decreased frequency of breathing and an increase in tidal volume. Another reusable alternative to N95 FFRs is the Powered AirPurifying respirator (PAPR). The physiological benefit of PAPRs is that they have a fan that blows fresh air through the filter; therefore, there should not be any sense of breathing resistance as experienced with an N95 FFR or an EHMR. However, there may be other psychophysiological effects resulting from the constant noise produced by the PAPR motor, such as headache, distraction, anxiety, difficulty communicating with others in the room to mention a few. ${ }^{[14]}$

The major limitation of this study was inability to evaluate the effects of N95 mask usage at higher work intensities and over longer durations because of ethical concerns. Sample size for the study was small thus future research is required with more sample size.

\section{CONCLUSION}

Prolonged use of N95 mask during COVID-19 has caused adverse effects such as reduction in blood oxygen saturation level. In preparation for future pandemics, it is imperative to identify solutions to manage these adverse effects. Scheduled breaks, improved hydration and rest and potentially newly designed comfortable masks are recommended for future management of adverse effects related to prolonged use of masks.

\section{Acknowledgement: None}

Conflict of Interest: None

\section{Source of Funding: None}

\section{Ethical Approval: Approved}

\section{REFERENCES}

1. Wu D, Wu T, Liu Q, Yang Z. The SARSCoV-2 outbreak: What we know. Int J Infect Dis. 2020 May;94:44-48

2. Burki $\mathrm{T}$, outbreak of corona virus disease 2019. Lancet infet dis 2019: s 1473-3099: 30076-30081

3. Guidance for Wearing Masks. Help Slow the Spread of COVID-19 [Internet] 2020 [Updated Feb. 18, 2021: cited 2021 March 11] Available from https://www.cdc.gov/coronavirus/2019ncov/prevent-getting-sick/cloth-face-coverguidance

4. World Health Organization. (2020). Advice on the use of masks in the context of COVID-19: interim guidance, 5 June 2020. World Health Organization.

5. Zhang M, Emery AR, Tannyhill RJ 3rd, Zheng H, Wang J. Masks or N95 Respirators During COVID-19 PandemicWhich One Should I Wear? J Oral Maxillofac Surg. 2020 Dec;78(12):21142127

6. N95 respirators and surgical masks (face masks). [Internet] 2020. [Updated April 2020. Accessed April 5, 2020] Available from https://www.fda.gov/medicaldevices/personalprotective-equipmentinfection-control/n95-respirators-andsurgical-masksface-masks

7. Rebmann T., Carrico R., Wang J. Physiologic and other effects and compliance with long-term respirator use among medical intensive care unit nurses. Am J Infect Control. 2013

8. Beder, A.; Buyukkoçak, U.; Sabuncuo glu, H.; Keskil, Z.A.; Keskil, S. Preliminary report on surgical mask induced deoxygenation during major surgery. Neurocirugía 2008, 19, 121-126

9. Eninger RM, Honda T, Adhikari A, Heinonen-Tanski H, Reponen T, Grinshpun SA. Filter performance of $\mathrm{n} 99$ and $\mathrm{n} 95$ facepiece respirators against viruses and ultrafine particles. Ann Occup Hyg. 2008; 52(5):385-396

10. Safety of Extended Use and Reuse of N95 Respirators. [Internet] March 2020. Available

from 
Rajesh Prabhakar Gaikwad et.al. Respiratory consequences of N95 mask during Covid-19 pandemic- an observational study.

https://www.elsevier.com/_data/assets/pdf _file/0006/997863/COVID-ECRI-N95-

Respirators_2020-03.pdf

11. Williams WJ. Physiological response to alterations in [O2] and [CO2]: relevance to respiratory protective devices. J Intl Soc Resp Protect 2010; 27(1):27-51

12. Tong, Pearl Shuang Ye; Kale, Anita Sugam; $\mathrm{Ng}$, Kailyn; Loke, Amelia Peiwen; Choolani, Mahesh Arjandas; Lim, Chin Leong; Chan, Yiong Huak; Chong, Yap Seng; Tambyah, Paul Anantharajah; Yong, Eu-Leong (2015). Respiratory consequences of N95-type Mask usage in pregnant healthcare workers- a controlled clinical study. Antimicrobial Resistance and Infection Control, 4(1), 48

13. Lim ECH, Seet RCS, Lee KH, WilderSmith EPV, Chuah BYS, Ong BKC. Headaches and the N95 face-mask amongst healthcare providers. Acta Neurologica Scandinavica. 2006;113(3):199-202

14. Jon Williams, Jaclyn Krah Cichowicz, Adam Hornbeck, Jonisha Pollard, Jeffrey
Snyder. The Physiological Burden of Prolonged PPE Use on Healthcare Workers during Long Shifts. [Internet] June 10, 2020. Available from https://blogs.cdc.gov/niosh-scienceblog/2020/06/10/ppe-burden/

15. Hines $S$, Brown $C$, Oliver $M$, Gucer $P$, Frisch M, Hogan R, Roth T, Chang J, McDiarmid M. User acceptance of reusable respirators in health care. Am J Infect Contr. 2019; 47:648-655

16. Wu S, Harber P, Yun D, Bansal S, Li Y, Santiago S. Anxiety during respirator use: Comparison of two respirator types. Journal of Occupational and Environmental Hygiene. 2011;8(3):123-128

How to cite this article: Gaikwad RP, Banodkar AB, Nandgaonkar VP et.al. Respiratory consequences of N95 mask during Covid-19 pandemic- an observational study. Int $J$ Health Sci Res. 2021; 11(4): 55-61. DOI: https://doi.org/ 10.52403/ijhsr.20210407 